\title{
How about actively using telemedicine during the COVID-19 pandemic?
}

\author{
Yong Sauk Hau ${ }^{1,2}$ • Jeoung Kun Kim ${ }^{1,2}$ • Jian Hur ${ }^{3} \cdot$ Min Cheol Chang ${ }^{2,4}$
}

Received: 14 April 2020 / Accepted: 23 April 2020 / Published online: 30 April 2020

(C) Springer Science+Business Media, LLC, part of Springer Nature 2020

Keywords Coronavirus disease $\cdot$ Geriatric population $\cdot$ Infection $\cdot$ Telemedicine $\cdot$ Mental health

To the editor:

The first case of coronavirus disease (COVID-19) occurred in Wuhan, China, in December 2019. Outbreaks have affected most countries in the world, with rapid dissemination via unprecedented propagation. The World Health Organization (WHO) declared COVID-19 as being pandemic on March 11, 2020. Although the fatality rate of COVID-19 is about 2-5\%, which is not very high, the fatality rate among the geriatric population aged over 60 years is greater than $10 \%$. The fatality rate is even higher for individuals in their 70s and 80s [1].

Many research studies have reported that the COVID-19 pandemic causes psychiatric problems, such as anxiety and depression $[2,3]$. As such, the geriatric population needs psychiatric counseling or treatment, but it is difficult for the geriatric population to visit hospitals or clinics because of the risk and fear of COVID-19 infection. Furthermore, with the current COVID-19 crisis showing signs of being prolonged, measures that address these problems are urgently needed.

Telemedicine, which is also used synonymously with "remote medical care," refers to providing clinical healthcare through electronic communication technologies rather than through inperson meetings between a patient and a doctor. ${ }^{4,5}$ The initial

This article is part of the Topical Collection on Mobile \&amp; Wireless Health

Min Cheol Chang

wheel633@ynu.ac.kr

1 Department of Business Administration, School of Business, Yeungnam University, Gyeongsan-si, Republic of Korea

2 Medical Management Research Center, School of Business, Yeungnam University, Taegu, Republic of Korea

3 Department of Infectious Disease Internal Medicine, College of Medicine, Yeungnam University, Taegu, Republic of Korea

4 Department of Physical Medicine and Rehabilitation, College of Medicine, Yeungnam University, Taegu, Republic of Korea form of telemedicine involved the use of a telephone. The use of video calls and other telecommunication applications more recently has improved the service greatly. Through the latest advances in technology, we believe that telemedicine can improve the mental and physical health of the geriatric population.

With telemedicine, patients who need care for anxiety and depression can be assisted without the requirement for visiting a hospital, and therapy for psychological stabilization can be provided via the internet, without the need for an in-person consultation with the doctor $[3,4]$. For the geriatric population, telemedicine consultations can also help with overcoming isolation and loneliness due to disconnection with the external world. Moreover, by reducing the number of hospital visits for periodical consultations and prescriptions among the geriatric population with mental illnesses, telemedicine may potentially reduce the number of secondary or tertiary infections that could occur on route to the hospital or while waiting for care. Furthermore, telemedicine may also reduce the loss to follow up among psychiatric patients. Through telemedicine, doctors are able to continuously identify and manage each patient's condition, which may prevent patients from not receiving appropriate treatment if their psychiatric symptoms worsen.

During the COVID-19 pandemic, if a viral infection is suspected in a geriatric patient, telemedicine can help the doctor triage the patient and discern whether a visit to the hospital for COVID-19 testing is warranted. Additionally, for geriatric patients diagnosed with COVID-19 who have minor or no symptoms and are quarantined at home, the doctor can continuously assess the patients' condition through telemedicine and ensure early detection of worsening symptoms to prevent missing the window of opportunity for treatment.

Telemedicine is also advantageous for geriatric populations located in regions far away from the city (that is, regions without sufficient hospitals and clinics) in terms of accessing appropriate and timely medical services in many areas, including psychiatry. 
The positive effects of psychiatric consultation and treatment through telemedicine have been verified in many previous studies $[4,5]$. In 2013, Hilty and colleagues analyzed studies on the effects of telemedicine published to date and reported that psychiatric consultation and treatment through telemedicine for people across many age groups, including the geriatric population, has an effect that is comparable to in-person care [4]. Furthermore, it was reported that there were effects across many settings, including emergency and home health care settings.

We have examined how telemedicine can benefit geriatric psychiatric health in the current COVID-19 pandemic. Given the high mortality rate among the geriatric population, this population is considered as being at the highest risk for COVID-19. There is also an increased probability of social withdrawal and isolation among the geriatric population in this situation, and as direct visits to hospitals are difficult, there are greater concerns about their mental health. It is expected that telemedicine will play a useful role in protecting the mental health of the geriatric population during the COVID-19 pandemic and in other future situations associated with outbreaks of infectious diseases.

Funding information The present study was supported by a National Research Foundation of Korea grand funded by the Korean government (grant no. NRF-2019M3E5D1A0268106).

\section{Compliance with Ethical Standards}

Conflicts of Interest The authors report no conflict of interest.

Human Participants and/or Animals This study does not contain any studies with human participants or animals.
Informed Consent Not applicable.

\section{References}

1. Kucharski, A. J., Russell, T. W., Diamond, C., Liu, Y., Edmunds, J., Funk, S., Eggo, R. M., and Centre for Mathematical Modelling of Infectious Diseases COVID-19 working group, Early dynamics of transmission and control of COVID-19: a mathematical modelling study. The Lancet. Infectious diseases, [ahead of print]. https://doi. org/10.1016/S1473-3099(20)30144-4, 2020

2. Nguyen, H. C., Nguyen, M. H., Do, B. N., Tran, C. Q., Nguyen, T., Pham, K. M., Pham, L. V., Tran, K. V., Duong, T. T., Tran, T. V., Duong, T. H., Nguyen, T. T., Nguyen, Q. H., Hoang, T. M., Nguyen, K. T., Pham, T., Yang, S. H., Chao, J. C., and Duong, T. V, People with Suspected COVID-19 Symptoms Were More Likely Depressed and Had Lower Health-Related Quality of Life: The Potential Benefit of Health Literacy. Journal of clinical medicine, 9(4);E965. https://doi.org/10.3390/jcm9040965, 2020

3. Torales, J., O'Higgins, M., Castaldelli-Maia, J.M., and Ventriglio, A, The outbreak of COVID-19 coronavirus and its impact on global mental health. Int J Soc Psychiatry, [ahead of print]. doi:https://doi. org/10.1177/0020764020915212, 2020

4. Hilty, D.M., Ferrer, D.C., Parish, M.B., Johnston, B., Callahan, E.J., and Yellowlees, P.M, The effectiveness of telemental health: a 2013 review. Telemed J E Health, 19(6):444-454, doi:https://doi.org/10. 1089/tmj.2013.0075, 2013.

5. Nair, U., Armfield, N. R., Chatfield, M. D., and Edirippulige, S, The effectiveness of telemedicine interventions to address maternal depression: A systematic review and meta-analysis. Journal of telemedicine and telecare, 24(10):639-650, https://doi.org/10.1177/ 1357633X18794332, 2018.

Publisher's note Springer Nature remains neutral with regard to jurisdictional claims in published maps and institutional affiliations. 